\title{
Edentulous people prefer lingualised or anatomic posterior occlusal tooth forms
}

\author{
What is the best posterior tooth form for complete dentures?
}

\author{
Sutton AF, Worthington HV, McCord JF. \\ RCT comparing posterior occlusal forms for complete dentures. $J$ \\ Dent Res 2007; 86:651-655
}

Design This was a randomised cross-over study.

Intervention Participants were randomly assigned to receive one of three sets of complete dentures with different types of posterior occlusal design forms, either anatomic teeth, lingualised teeth, and zero-degree teeth.

Outcome measure The primary outcome measure was a visual analogue scale (VAS) focusing on five aspects of denture satisfaction. Data were recorded at baseline, considering their original dentures, and 8 weeks post-final review.

Results The chewing function of the complete dentures provided with lingualised and anatomic posterior occlusal forms was preferred, statistically significantly, over that of zero-degree posterior occlusal forms ( $P 0.004$ and $P 0.001$, respectively).

Conclusions The findings of this study suggest that the participants significantly preferred posterior occlusal schemes with anatomic and lingualised teeth, compared with cuspless teeth.

\section{Commentary}

This was a classic randomised controlled trial (RCT) based on an excellent idea but with less-than-perfect execution. The primary objective was clearly and plainly stated: to test the null hypothesis that there is no variation in the level of individual satisfaction for three different types of posterior occlusal forms for complete dentures. Being published in a research-oriented journal, it is understandable that the objective was stated using such statistical wording rather than more clinical language.

The inclusion criteria for the 50 eligible participants were not properly formulated. In order to be eligible for inclusion in this study, one only had to be edentulous, free from any debilitating diseases or oral pathologies, and psychologically stable. The age range of the participants was not set for the study. In addition, how long people had been edentulous prior to the study was not considered and therefore neither was the degree of bone resorption, a factor that may have affected the level of patient satisfaction regardless of the occlusal form. The study was also not designed in a way that guarantees an equal gender distribution because, at the end of the study, there turned out to be twice as many female as male participants. Furthermore, the results of this study were based on 41 participants although 45 individuals participated in the trial. The lack of intention-to-treat analysis may have affected the results of the study.
The authors used a VAS to measure patients' satisfaction with the dentures. The VAS has long been established as an instrument for measuring general satisfaction with oral clinical interventions and is a good instrument for the purpose of the trial since it can measure changes in attitudes within individuals. Ironically, the authors reported the use of a completely different tool for the assessment of patient satisfaction, namely the Oral Health Impact Profile20 EDENT (OHIP-EDENT), ${ }^{1,2}$ when the same study was published somewhere else. ${ }^{3}$

On the positive side, the randomisation process was described in detail and blinding was done to the maximum that the design of the study permits.

With some reservations, this RCT may be considered the first step in building a body of evidence of reasonable quality that favours the use of lingualised and anatomic posterior occlusal forms over the zero-degree form with regards to the satisfaction of complete-denture patients. It is quite shocking to realise the huge lack of good-quality evidence in this area. Even the results of the only available systematic review on this topic were disappointing, ${ }^{4}$ with only one RCT of poor quality included. Because of this lack of evidence, it is apparent that the choice of the occlusal form has been based on the operator's experience and/ or patient preference rather than evidence. The existence of this RCT at least means this choice will be more likely to be based on some sort of evidence.

\section{Practice point}

Patients tend to prefer lingualised or anatomic posterior occlusal forms over the zero degree form.

\section{Asim Al-Ansari \\ Dental Department, Armed Forces Hospital, Dhahran, \\ Saudi Arabia \\ 1. Allen PF. Assessment of oral health related quality of life. Health Qual Life Outcome 2003; 1:40. \\ 2. Allen F, Locker D. A modified short version of the oral health impact profile for assessing health-related quality of life in edentulous adults. Int J Prosthodont 2002; 15:446-450. \\ 3. Sutton AF, McCord JF. A randomised clinical trial comparing anatomic, lingualised, and zero-degree posterior occlusal forms for complete dentures. J Prosthet Dent 2007; 97:292-298. \\ 4. Sutton AF, Glenny AM, McCord JF. Interventions for replacing missing teeth: denture chewing surface designs in edentulous people. Cochrane Database Syst Rev 2005; issue 1.}

Evidence-Based Dentistry (2007) 8, 113. doi:10.1038/sj.ebd.6400530 